\title{
Divergências metodológicas entre os estudos que avaliaram a associação entre as síndromes hipertensivas da gestação e a prematuridade: uma revisão integrativa da
}

\section{literatura}

\author{
Methodological differences between studies that evaluated the association between hypertensive \\ syndromes of pregnancy and prematurity: an integrative literature review
}

Diferencias metodológicas entre los estudios que evaluaron la asociación entre los síndromes hipertensivos del embarazo y la prematuridad: una revisión integrativa de la literatura

Recebido: 14/09/2021 | Revisado: 26/09/2021 | Aceito: 30/09/2021 | Publicado: 02/10/2021

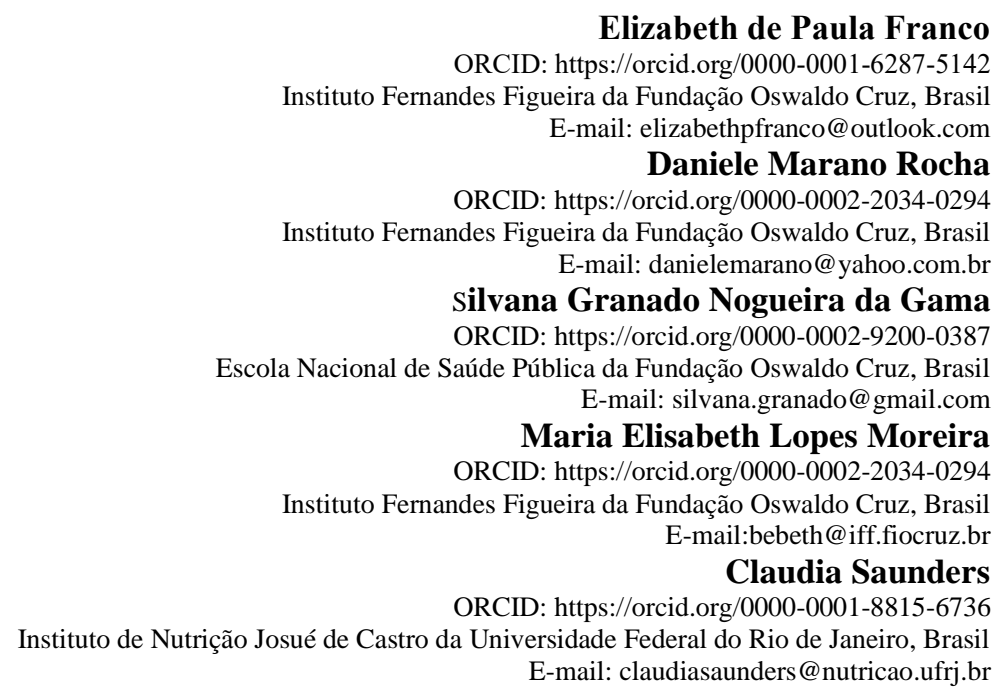

\begin{abstract}
Resumo
Objetivos: Avaliar as divergências metodológicas entre os estudos que analisaram a associação entre as síndromes hipertensivas da gestação e a prematuridade. Métodos: Trata-se de em uma revisão integrativa da literatura, realizada entre setembro de 2020 e janeiro de 2021. A busca dos artigos foi realizada nas bases de dados PubMed, Biblioteca Virtual em Saúde, Embase, Web of Science e Scopus com base na combinação dos seguintes descritores: ("Pregnancy Induced Hypertension" OR "High Risk Pregnancy") AND ("Prematurity" OR "Prematury Birth" OR "Premature Neonate" OR "Neonate Prematurity"). Resultados e Discussão: Foram encontrados 582 artigos, sendo apenas nove foram selecionados. As principais disparidades metodológicas observadas foram relativas à classificação das síndromes hipertensivas da gestação e da prematuridade, idade das participantes e controle de confundidores. Do total de estudos selecionados, cinco informaram as diferentes diretrizes utilizadas para a classificação das síndromes e seis utilizaram o ponto de corte inferior a 37 semanas para a prematuridade. A maior parte dos artigos encontraram associação entre as diferentes categorias das síndromes hipertensivas e a prematuridade. Conclusão: Mesmo diante das divergências metodológicas observadas entre os estudos, foi verificada associação entre as síndromes hipertensivas da gestação e a prematuridade. Para fins comparativos, é recomendável que novos estudos utilizem as diretrizes internacionais vigentes para definição das síndromes hipertensivas e da prematuridade. Além disso, é importante ressaltar a importância da adequação do pré-natal com intuito de acompanhar e tratar as mulheres com síndromes hipertensivas visando a diminuição das complicações decorrentes desta condição clínica, sobretudo a prematuridade.
\end{abstract}

Palavras-chave: Hipertensão induzida pela gestação; Prematuridade; Neonatos; Revisão integrativa da literatura.

\footnotetext{
Abstract

Objectives: To assess methodological differences between studies that analyzed the association between hypertensive syndromes of pregnancy and prematurity. Methods: This is an integrative literature review, carried out between September 2020 and January 2021. The search for articles was performed in the PubMed, Virtual Health Library, Embase, Web of Science and Scopus databases based on combination of the following descriptors: ("Pregnancy Induced
} 
Hypertension" OR "High Risk Pregnancy") AND (“Prematurity” OR "Prematury Birth" OR "Premature Neonate" OR "Neonate Prematurity"). Results and Discussion: 582 articles were found, only nine were selected. The main methodological disparities observed were related to the classification of hypertensive syndromes of pregnancy and prematurity, age of participants and control of confounders. Of the total number of selected studies, five informed the different guidelines used for the classification of syndromes and six used a cutoff point below 37 weeks for prematurity. Most articles found an association between the different categories of hypertensive syndromes and prematurity. Conclusion: Despite the methodological differences observed between the studies, an association was found between hypertensive syndromes of pregnancy and prematurity. For comparative purposes, it is recommended that further studies use current international guidelines to define hypertensive syndromes and prematurity. In addition, it is important to emphasize the importance of adequate prenatal care in order to monitor and treat women with hypertensive syndromes in order to reduce complications resulting from this clinical condition, especially prematurity.

Keywords: Pregnancy-induced hypertension; Prematurity; Neonates; Integrative literature review.

\section{Resumen}

Objetivos: Evaluar las diferencias metodológicas entre los estudios que analizaron la asociación entre los síndromes hipertensivos del embarazo y la prematuridad. Métodos: Se trata de una revisión integrativa de la literatura, realizada entre septiembre de 2020 y enero de 2021. La búsqueda de artículos se realizó en las bases de datos PubMed, Virtual Health Library, Embase, Web of Science y Scopus en base a la combinación de los siguientes descriptores: ("Pregnancy Induced Hypertension" OR "High Risk Pregnancy") AND ("Prematurity" OR "Prematury Birth" OR "Premature Neonate" OR "Neonate Prematurity"). Resultados y Discusión: Se encontraron 582 artículos, solo se seleccionaron nueve. Las principales disparidades metodológicas observadas se relacionaron con la clasificación de los síndromes hipertensivos del embarazo y la prematuridad, la edad de las participantes y el control de los factores de confusión. Del número total de estudios seleccionados, cinco informaron las diferentes pautas utilizadas para la clasificación de síndromes y seis utilizaron un punto de corte por debajo de 37 semanas para la prematuridad. La mayoría de los artículos encontraron una asociación entre las diferentes categorías de síndromes hipertensivos y prematuridad. Conclusión: A pesar de las diferencias metodológicas observadas entre los estudios, se encontró asociación entre los síndromes hipertensivos del embarazo y la prematuridad. Con fines comparativos, se recomienda que los estudios adicionales utilicen las guías internacionales actuales para definir los síndromes hipertensivos y la prematuridad. Además, es importante enfatizar la importancia de una atención prenatal adecuada para monitorear y tratar a las mujeres con síndromes hipertensivos con el fin de reducir las complicaciones derivadas de esta condición clínica, especialmente la prematuridad.

Palabras clave: Hipertensión inducida por el embarazo; Precocidad; Recién nacidos; Revisión integrativa de la literatura.

\section{Introdução}

A gestação é um fenômeno fisiológico e sua evolução, na maioria dos casos, segue sem intercorrências (Oliveira et al., 2016; Antunes et al., 2017). Contudo, as síndromes hipertensivas da gestação (SHG) são as complicações mais comuns nesse período, incidindo em até 10\% das gestantes (OMS, 2014; Braunthal \& Brateanu, 2019).

No cenário da saúde pública mundial, as SHG merecem atenção especial por se constituírem uma das principais causas de mortalidade materna e perinatal (Braunthal \& Brateanu, 2019; Shah \& Gupta, 2019). Em países desenvolvidos, como os Estados Unidos, as SHG contribuem com 7\% a 12\% na mortalidade materna (Shah \& Gupta, 2019), na África e na Ásia incidem em 9\%, enquanto nos países da América Latina e Caribe, em 26\% (ACOG, 2019).

Além de acarretar o aumento da mortalidade materna, as SHG estão associadas a uma série de desfechos adversos, tais como: o baixo peso ao nascer (Antunes et al., 2017), a restrição de crescimento intrauterino (Lima et al., 2018), o deslocamento de placenta (Sousa et al., 2019), a prematuridade (Cruz et al., 2018), o óbito fetal e neonatal (Morais et al., 2013), entre outros.

Dentre os desfechos aludidos, a prematuridade é considerada uma complicação frequente das SHG (Viana et al., 2014; Pereira et al., 2018; Scime et al., 2020), seja pela ocorrência do trabalho de parto prematuro espontâneo, em virtude das contrações uterinas aumentadas, ou pela conduta obstétrica de interrupção da gestação em razão das condições maternas e/ou fetais desfavoráveis (Cruz et al., 2018; Scime et al., 2020; Brandi et al., 2020).

De acordo com o Relatório de Ação Global sobre Prematuridade, em 2010, dos 135 milhões de recém-nascidos vivos em todo o mundo, 11,1\% nasceram prematuros (Blencowe et al., 2013). Esse desfecho é considerado um desafio para a saúde pública e representa a principal causa de morbimortalidade neonatal, podendo acarretar sequelas imediatas e tardias, desde as 
complicações respiratórias, enterocolite necrosante, hipotermia, hipoglicemia, hiperbilirrubinemia, doença pulmonar crônica e paralisia cerebral. Além de gerar altos custos econômicos para o sistema de saúde e para as famílias (Chaim et al., 2008; Purisch \& Gyamfi-Bannerman, 2017; Cruz et al., 2018)

Inúmeros artigos já avaliaram a associação entre as SHG e a prematuridade (Barton et al., 2011; Lu et al., 2018; Cruz et al., 2018), no entanto, ainda não há consenso na literatura. Diante do exposto, o presente estudo objetivou verificar as divergências metodológicas entre os artigos que avaliaram a associação entre as SHG e a prematuridade.

\section{Metodologia}

A metodologia utilizada para elaboração deste trabalho foi uma revisão integrativa da literatura. Esse tipo de revisão propõe síntese do conhecimento e o agrupamento dos resultados de estudos relevantes permitindo a fundamentação da prática sobre determinado tema ou questão com base em evidências científicas segundo Sousa et al. (2017).

Foi realizada uma busca de artigos que avaliaram a associação entre as SHG e a prematuridade, com base na descrição embasada na diretriz Prefered Reporting Itens for Systematic Reviews (PRISMA) (Moher et al., 2009).

A definição da questão da pesquisa foi estruturada por meio do acrônimo PECO, em que cada letra equivale a um componente da pergunta condutora: (P) População: gestantes; (E) Exposição: diagnóstico de SHG; (C) Controle (não expostos): gestantes sem diagnóstico de SHG; (O) Outcome (desfecho): prematuridade (Brasil, 2014).

Após a determinação da questão de pesquisa, foi conduzida a busca nas bases de dados PubMed, Biblioteca Virtual em Saúde (BVS Brasil), Embase, Web of Science e Scopus, entre setembro de 2020 e janeiro de 2021. Foi utilizada a seguinte combinação de descritores para busca dos artigos: ("Pregnancy Induced Hypertension" OR "High Risk Pregnancy") AND ("Prematurity" OR "Prematury Birth" OR "Premature Neonate" OR "Neonate Prematurity") que foram modificados segundo as regras de indexação de cada base de dados. Com o intuito de ampliar a busca bibliográfica, não houve utilização de filtro em relação ao tipo de estudo, a data de publicação e o idioma.

Os artigos foram avaliados de forma independente por dois pesquisadores. A primeira etapa de seleção dos artigos consistiu na leitura dos títulos e dos resumos para identificação e remoção de estudos que não atenderam ao objetivo da presente revisão integrativa da literatura. Todos os artigos que passaram por esse filtro foram lidos na íntegra para aplicação dos critérios de elegibilidade.

Foram considerados elegíveis os estudos que avaliaram gestantes diagnosticadas com SHG e que tiveram a prematuridade como um dos desfechos avaliados. Os critérios de exclusão adotados foram artigos que verificaram a associação entre as SHG e outros desfechos descritos a seguir: retinopatia da prematuridade, disfunção hepática, fertilização in vitro, tipo de parto, diabetes mellitus e obesidade materna, doença renal crônica, marcadores inflamatórios, hiperucemia, hemoconcentração, amamentação, alteração do fluxo sanguíneo e corioamionite. Todos os estudos foram armazenados no programa Zotero para organização das referências.

Os dados dos artigos selecionados foram tabulados de acordo com os seguintes critérios: autor, ano, país/cidade, tamanho da amostra, delineamento do estudo, idade materna em anos, categorias avaliadas das SHG, diretrizes para a classificação das SHG, idade gestacional no nascimento, critérios de elegibilidade, critérios de exclusão, fatores de confusão controlados na análise, perdas do estudo e principais resultados observados.

\section{Resultados}

A estratégia de busca estabelecida resultou em 582 artigos, sendo 82 no PubMed, 219 na BVS, 137 no Embase, 14 na Web of Science e 130 no SCOPUS. Após a leitura dos títulos e resumos, foram excluídos 518 artigos, restando 64 para serem 
lidos integralmente. Desse total, 53 artigos foram excluídos, resultando nove estudos para compor a presente revisão integrativa. O fluxograma com o processo de busca e seleção dos artigos se encontra na Figura 1.

Figura 1: Fluxograma do processo de seleção dos artigos selecionados.

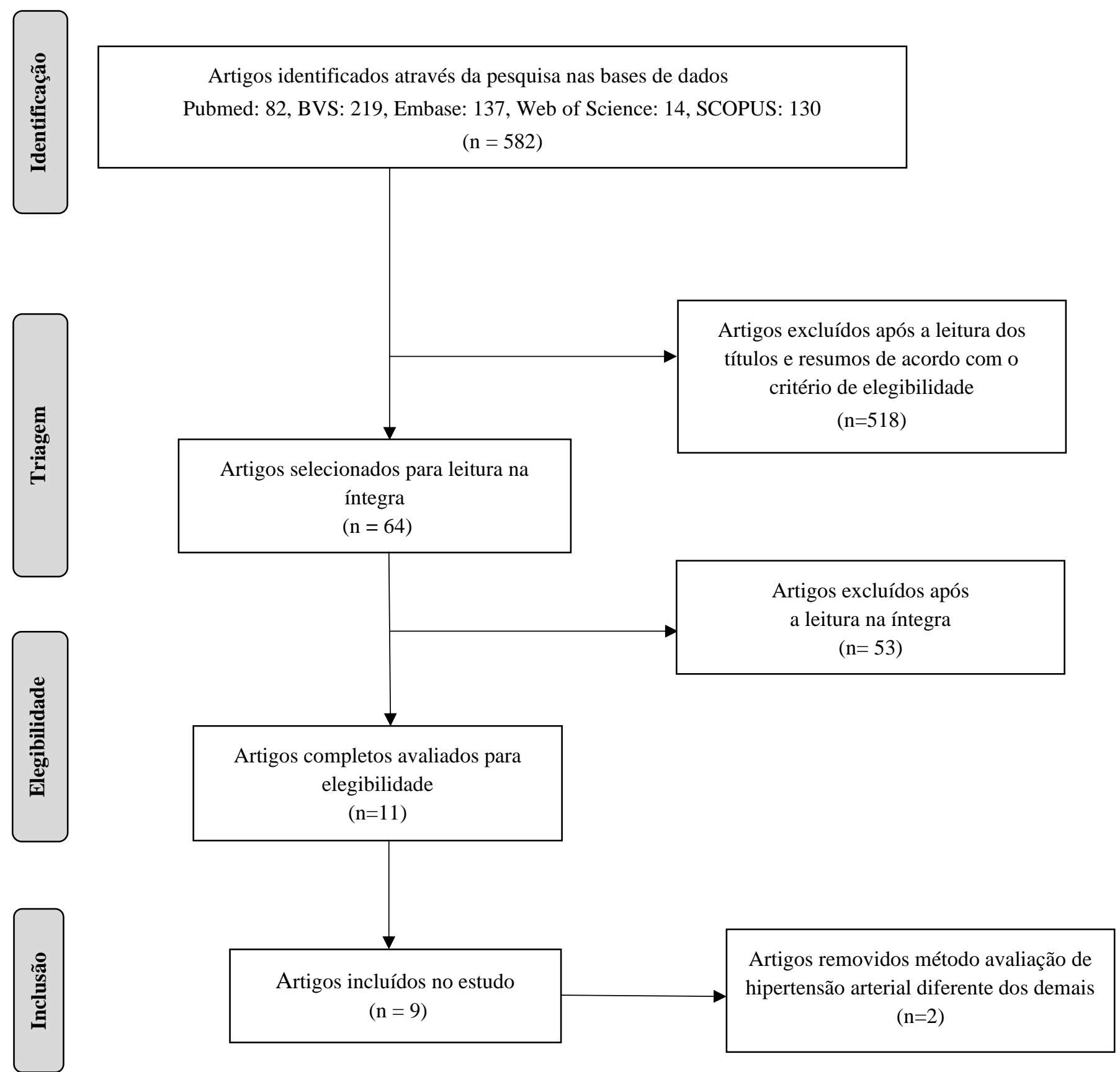

Fonte: Autores.

Foram identificados, seis artigos, no período de 2005 e 2010, e três, entre 2014 e 2019 . A respeito do local dos estudos, dois foram realizados na Austrália, dois na Espanha, dois na África, um no Paquistão e dois no Brasil. O tamanho amostral dos estudos foi de 12 a 255.931 participantes. Quanto ao delineamento dos estudos selecionados, seis foram transversais, dois casocontrole e uma coorte. Sobre a idade materna, dois apresentaram apenas a média da idade, um utilizou intervalo interquartil (16 a 41 anos) e a média, um a mediana, um avaliou mulheres com idade inferior a 20 anos e mulheres com idade superior a 35 anos e quatro estudos não informaram a faixa etária das participantes, como apresentados no Quadro 1. 
Quadro 1. Descrição dos estudos quanto aos autores, país e cidade do estudo, tamanho da amostra, delineamento do estudo, idade materna, 2005 a 2019.

\begin{tabular}{|c|c|c|c|c|}
\hline Autores e ano & País e cidade do estudo & Tamanho da amostra & $\begin{array}{l}\text { Delineamento } \\
\text { do estudo }\end{array}$ & $\begin{array}{l}\text { Idade materna } \\
\text { (anos) }\end{array}$ \\
\hline $\begin{array}{l}\text { Roberts et al. } \\
(2005)\end{array}$ & Austrália (Sydney) & $\begin{array}{c}\text { Total de gestantes: } 250.173 \\
\text { Gestantes hipertensas: } 24.517 \\
\text { Recém-nascidos: } 255.931\end{array}$ & Transversal & $<20 ; 20$ a $34 ; \geq 35$ \\
\hline $\begin{array}{l}\text { Oliveira et al. } \\
(2006)\end{array}$ & Brasil (Recife) & $\begin{array}{c}\text { Total de gestantes: } 12.272 \\
\text { Gestantes controle: } 11.013 \\
\text { Gestantes hipertensas: } 1.259\end{array}$ & Transversal & Não informada \\
\hline $\begin{array}{l}\text { Onyiriuka \& Okolo } \\
\text { (2007) }\end{array}$ & África (Nigéria) & $\begin{array}{c}\text { Gestantes caso: } 256 \\
\text { Gestantes controle: } 804\end{array}$ & Caso-controle & Não informada \\
\hline $\begin{array}{l}\text { Homer et al. } \\
(2008)\end{array}$ & Austrália (Sydney) & $\begin{array}{l}2540 \text { ((1192) Gestantes com } \\
\text { hipertensão gestacional, (1348) } \\
\text { pré-eclâmpsia com e sem } \\
\text { proteinúria) }\end{array}$ & Coorte & $\begin{array}{c}29,2 \pm 5,3(\text { média } \pm \\
\text { DP }) \\
30 \pm 4,7(\text { média } \pm \text { DP })\end{array}$ \\
\hline $\begin{array}{l}\text { Rojas Feria et al. } \\
(2009)\end{array}$ & Espanha (Sevilha) & $\begin{array}{l}\text { Total de gestantes: } 99 \\
\text { Recém-nascidos: } 12\end{array}$ & Transversal & $\begin{array}{c}30 \pm 5 \text { (média } \pm \text { DP }) \\
16-41 \\
\text { (intervalo } \\
\text { interquartílico) }\end{array}$ \\
\hline Mora et al. (2010) & Espanha (Valladolid) & Gestantes hipertensas: 65 & Transversal & Não informada \\
\hline $\begin{array}{l}\text { Bokhari et al. } \\
(2014)\end{array}$ & Paquistão (Lahore) & $\begin{array}{c}\text { Gestantes casos: } 50 \\
\text { Gestantes controle: } 50\end{array}$ & Caso-controle & Não informada \\
\hline Muti et al. (2015) & África (Harare) & $\begin{array}{c}\text { Gestantes normotensas:233 } \\
\text { Gestantes hipertensas: } 56\end{array}$ & Transversal & $\begin{array}{l}29 \text { (mediana) } \\
25 \text { (mediana) }\end{array}$ \\
\hline $\begin{array}{l}\text { Cassiano et al. } \\
\text { (2019) }\end{array}$ & $\begin{array}{l}\text { Brasil (Rio Grande do } \\
\text { Norte) }\end{array}$ & $\begin{array}{c}\text { Gestantes com pré-eclâmpsia } \\
\text { grave: } 157\end{array}$ & Transversal & $27 \pm 7$ (média \pm DP $)$ \\
\hline
\end{tabular}

DP: desvio-padrão. Fonte: Autores.

As diretrizes utilizadas para classificar as SHG foram informadas por cinco artigos. Quanto à classificação da prematuridade, seis estudos utilizaram a definição inferior a 37 semanas gestacionais. Dois estudos classificaram a prematuridade com base em diferentes categorias, a saber: <28; 28 a 32 e 33 a 36 semanas gestacionais e 20 a 32 e 33 a 36 semanas gestacionais e um artigo não evidenciou o ponto de corte para classificação da prematuridade (Quadro 2). 


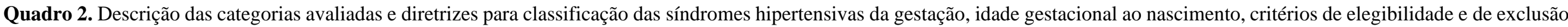
dos estudos incluídos no período de 2005 a 2019

\begin{tabular}{|c|c|c|c|c|c|}
\hline Autores e ano & $\begin{array}{c}\text { Categorias avaliadas das } \\
\text { síndromes hipertensivas da } \\
\text { gestação } \\
\end{array}$ & $\begin{array}{c}\text { Diretrizes para } \\
\text { classificação das síndromes } \\
\text { hipertensivas da gestação } \\
\end{array}$ & $\begin{array}{c}\text { Idade gestacional } \\
\text { ao nascimento } \\
\text { (semanas) } \\
\end{array}$ & Critérios de elegibilidade & Critérios de exclusão \\
\hline Roberts et al. (2005) & $\begin{array}{c}\text { Hipertensão crônica } \\
\text { Pré-eclâmpsia/eclâmpsia } \\
\text { Hipertensão crônica sobreposta } \\
\text { a pré-eclâmpsia } \\
\text { Hipertensão gestacional }\end{array}$ & (CID 10 - 10ed) & $\begin{array}{l}20 \text { a } 32 \\
33 \text { a } 36\end{array}$ & $\begin{array}{c}\text { Recém-nascidos com } 20 \text { semanas } \\
\text { gestacionais ou } 400 \mathrm{~g} \text { de peso ao nascer e } \\
\text { diagnóstico positivo para hipertensão } \\
\text { materna }\end{array}$ & Não informado \\
\hline Oliveira et al. (2006) & $\begin{array}{l}\text { Grupo } 1 \text { (gestantes com } \\
\text { hipertensão com e sem } \\
\text { proteinúria) } \\
\text { Grupo } 2 \text { (gestantes com } \\
\text { hipertensão crônica) } \\
\text { Grupo } 3 \text { (gestantes } \\
\text { normotensas) }\end{array}$ & Não informado & $<37$ & $\begin{array}{l}\text { Gestantes com hipertensão com pressão } \\
\text { arterial } \geq 140 \text { x } 90 \mathrm{mmHg} \text {. Parto de } \\
\text { concepto com idade gestacional maior } \\
\text { que } 20 \text { semanas e gestação única }\end{array}$ & Não informado \\
\hline Onyiriuka \& Okolo (2007) & $\begin{array}{l}\text { Grupo caso (recém-nascidos de } \\
\text { gestantes com hipertensão } \\
\text { transitória, pré-eclâmpsia } \\
\text { severa, eclâmpsia, hipertensão } \\
\text { crônica e hipertensão crônica } \\
\text { sobreposta à pré-eclâmpsia) } \\
\text { Grupo controle (recém-nascidos } \\
\text { de gestantes normotensas) }\end{array}$ & $\begin{array}{c}\text { (Welt \& Crenshaw, 1978; } \\
\text { Acien P, 1986; Cunningham } \\
\text { et al., 1993) }\end{array}$ & $<37$ & $\begin{array}{l}\text { Grupo caso: Histórico de hipertensão } \\
\text { antes da gestação; aumento da pressão } \\
\text { arterial sistólico ou diastólica superior de } \\
15 \text { ou 30mmHg respectivamente; pressão } \\
\text { arterial intraparto de } 140 / 90 \mathrm{mmHg} \text { em } \\
\text { pelo menos duas ocasiões com intervalo } \\
\text { mínimo de } 6 \text { horas durante o parto. } \\
\text { Grupo controle: mulheres normotensas } \\
\text { durante toda a gestação, na admissão para } \\
\text { o parto, sem exposição conhecida a } \\
\text { sedativos, diuréticos e medicamentos } \\
\text { anti-hipertensivos }\end{array}$ & $\begin{array}{c}\text { Ambos os grupos de (mães): } \\
\text { não fumantes, sem doenças } \\
\text { como diabetes mellitus, } \\
\text { anemia falciforme, } \\
\text { insuficiência renal, doença } \\
\text { cardíaca, asma brônquica e } \\
\text { gestação gemelares. Ambos } \\
\text { os grupos: tiveram } \\
\text { isoimunização para Rhesus e } \\
\text { anomalias congênitas }\end{array}$ \\
\hline Homer et al. (2008) & $\begin{array}{c}\text { Hipertensão gestacional } \\
\text { Pré-eclâmpsia com proteinúria } \\
\text { Pré-eclâmpsia sem proteinúria }\end{array}$ & $\begin{array}{l}\text { Sociedade Internacional de } \\
\text { Estudos de Hipertensão na } \\
\text { Gestação } \\
\text { (Brown et al., 2001) }\end{array}$ & $<37$ & $\begin{array}{l}\text { Hipertensão sem solução após repouso } \\
\text { durante a noite no hospital ou altas } \\
\text { sucessivas após um dia de medicação } \\
\text { presença de proteinúria, fatores } \\
\text { neurológicos e bioquímicos anormais } \\
\text { (níveis séricos elevados de transaminase e } \\
\text { creatinina ou trombocitopenia, } \\
\text { internações recorrentes por hipertensão, } \\
\text { suspeita de causa secundária de } \\
\text { hipertensão) }\end{array}$ & Não informado \\
\hline Rojas Feria et al. (2009) & Síndrome HELLP & Martin et al.1999 & Não informou & $\begin{array}{c}\text { Plaquetas } \geq 150.000 \text { células } / \mathrm{m}, \text { disfunção } \\
\text { hepática (transaminases TGO, TGP ou } \\
\text { ambas } \geq 40 \mathrm{U} / \mathrm{L}, \text { hemólise, anemia } \\
\text { associada à presença de marcadores }\end{array}$ & Não informado \\
\hline
\end{tabular}


Research, Society and Development, v. 10, n. 12, e591101220591, 2021

(CC BY 4.0) | ISSN 2525-3409 | DOI: http://dx.doi.org/10.33448/rsd-v10i12.20591

\begin{tabular}{|c|c|c|c|c|c|}
\hline & & & & $\begin{array}{l}\text { indiretos de hemólise; } \mathrm{LDH} \geq 600 \mathrm{U} / \mathrm{L} \text { ou } \\
\text { aumento de bilirrubina }>1,2 \mathrm{mg} / \mathrm{dL} \text { ) }\end{array}$ & \\
\hline Mora et al. (2010) & $\begin{array}{c}\text { Hipertensão gestacional } \\
\text { Pré-eclâmpsia leve-moderada } \\
\text { Pré-eclâmpsia grave: } \\
\text { Eclâmpsia } \\
\text { Hipertensão crônica } \\
\text { Pré-eclâmpsia sobreposta à } \\
\text { hipertensão crônica }\end{array}$ & $\begin{array}{c}\text { Programa Nacional de } \\
\text { Educação sobre Pressão Alta } \\
\text { na Gestação (NHBPEP, 2000) } \\
\text { Estados Hipertensivos da } \\
\text { Gestação da Sociedade } \\
\text { Espanhola de Ginecologia e } \\
\text { Obstetrícia (SEGO, 1988) }\end{array}$ & $\begin{array}{c}<28 \\
28 \text { a } 32 \\
33 \text { a } 36\end{array}$ & $\begin{array}{c}\text { Hipertensão após } 20 \text { semanas } \\
\text { gestacionais associada à proteinúria } \\
\text { significativa }\end{array}$ & Não informado \\
\hline Bokhari et al. (2014) & $\begin{array}{l}\text { Grupo de gestantes pré- } \\
\text { eclâmpsia } \\
\text { Grupo de gestantes } \\
\text { normotensas }\end{array}$ & Não informado & $<37$ & $\begin{array}{c}\text { Grupo de gestantes com pré-eclâmpsia: } \\
\text { pressão arterial diastólica entre } 86 \text { a } 130 \\
\text { mmHg, edema e proteinúria. } \\
\text { Grupo de gestantes normotensas: } \\
\text { gestantes com pressão arterial diastólica } \\
\text { entre } 70 \text { e } 85 \text { mm Hg sem edema e } \\
\text { proteinúria } \\
\end{array}$ & Não informado \\
\hline Muti et al. (2015) & $\begin{array}{l}\text { Hipertensão gestacional } \\
\text { Gestantes normotensas }\end{array}$ & Não informado & $<37$ & $\begin{array}{l}\text { Mulheres que procuraram as } \\
\text { maternidades de Harare selecionadas, } \\
\text { residentes de Harare }\end{array}$ & Não informado \\
\hline Cassiano et al. (2019) & Pré-eclâmpsia grave & Não informado & $<37$ & $\begin{array}{l}\text { Fetos e neonatos de gestantes com } \\
\text { diagnóstico de PEG admitidas na } \\
\text { Unidade de Terapia Intensiva para } \\
\text { tratamento da síndrome hipertensiva e } \\
\text { que tiveram gravidez única, com parto } \\
\text { ocorrido entre } 23 \text { e } 42 \text { semanas de } \\
\text { gestação, independente da via e da } \\
\text { vitalidade fetal }\end{array}$ & Não informado \\
\hline
\end{tabular}

HELLP: H; (hemolysis); EL: (elevated liver); LP (low platelets). Fonte: Autores. 
Apenas um estudo realizou o controle de confundimento. No que tange as perdas, apenas dois as informaram (Quadro 3).

Quadro 3. Fatores de confusão controlados na análise, perdas e principais resultados dos estudos incluídos no período de 2005 a 2019

\begin{tabular}{|c|c|c|c|}
\hline Autores e ano & $\begin{array}{c}\text { Fatores de } \\
\text { confusão } \\
\text { controlados na } \\
\text { análise }\end{array}$ & Perdas & Principais resultados observados \\
\hline $\begin{array}{l}\text { Roberts et al. } \\
(2005)\end{array}$ & Não informado & Não se aplica & $\begin{array}{l}\text { O nascimento prematuro foi observado em todos os } \\
\text { estágios da síndrome hipertensiva da gestação analisadas }\end{array}$ \\
\hline $\begin{array}{l}\text { Oliveira et al. } \\
(2006)\end{array}$ & Não informado & Não se aplica & $\begin{array}{l}\text { O risco relativo de prematuridade foi maior no grupo com } \\
\text { hipertensão crônica em relação ao grupo com hipertensão } \\
\text { gestacional }\end{array}$ \\
\hline $\begin{array}{l}\text { Onyiriuka \& } \\
\text { Okolo (2007) }\end{array}$ & Não informado & $\begin{array}{l}\text { Recém-nascidos - mãe } \\
\text { hipertensa: } 16 \\
\text { Recém-nascidos - mãe } \\
\text { normotensa: } 12\end{array}$ & $\begin{array}{c}\text { Houve maior número de partos prematuros no grupo de } \\
\text { gestantes hipertensas em relação ao grupo de gestantes } \\
\text { normotensas }\end{array}$ \\
\hline $\begin{array}{l}\text { Homer et al. } \\
(2008)\end{array}$ & Paridade & 33 & $\begin{array}{l}\text { Mulheres que tiveram pré-eclâmpsia com proteinúria } \\
\text { foram mais propensas a terem recém-nascidos } \\
\text { prematuros em relação às mulheres com pré-eclâmpsia } \\
\text { sem proteinúria } \\
\text { O número de nascimentos prematuros foi } \\
\text { significativamente maior no grupo pré-eclâmpsia sem } \\
\text { proteinúria em relação ao grupo com hipertensão } \\
\text { gestacional }\end{array}$ \\
\hline $\begin{array}{l}\text { Rojas Feria et } \\
\text { al. (2009) }\end{array}$ & Não informado & Não se aplica & $\begin{array}{l}\text { A síndrome HELLP esteve relacionada com grande parte } \\
\text { dos nascimentos prematuros }\end{array}$ \\
\hline $\begin{array}{l}\text { Mora et al. } \\
(2010)\end{array}$ & Não informado & Não se aplica & $\begin{array}{l}\text { A prevalência de nascimentos prematuros foi maior entre } \\
\text { as mulheres com pré-eclâmpsia/eclâmpsia }\end{array}$ \\
\hline $\begin{array}{l}\text { Bokhari et al. } \\
\text { (2014) }\end{array}$ & Não informado & Não informado & $\begin{array}{c}\text { Houve um maior número de nascimentos prematuros no } \\
\text { grupo com PE em relação ao grupo de gestantes } \\
\text { normotensas }\end{array}$ \\
\hline $\begin{array}{l}\text { Muti et al. } \\
(2015)\end{array}$ & Não informado & Não se aplica & $\begin{array}{c}\text { Não houve diferença significativa de nascimentos } \\
\text { prematuros entre as mulheres com ou sem hipertensão } \\
\text { gestacional }\end{array}$ \\
\hline $\begin{array}{l}\text { Cassiano et al. } \\
(2019)\end{array}$ & Não informado & Não se aplica & $\begin{array}{l}\text { Dos } 157 \text { neonatos de gestantes com pré-eclâmpsia grave, } \\
48 \% \text { nasceram prematuros }\end{array}$ \\
\hline
\end{tabular}

Hellp: H; (hemolysis); EL: (elevated liver); LP (low platelets). Fonte: Autores.

Ao analisar a associação entre as categorias das SHG e a prematuridade (Quadro 3), Roberts et al. (2005) verificaram associação entre todas as categorias das SHG e a prematuridade. Mora et al. (2010) observaram a associação entre a préeclâmpsia/eclâmpsia e a prematuridade, enquanto Oliveira et al. (2006) identificaram risco superior para a prematuridade entre as mulheres com hipertensão crônica em relação àquelas com hipertensão gestacional. Homer et al. (2008) relataram maior número de nascimentos prematuros entre gestantes com PE sem proteinúria em relação às mulheres com hipertensão gestacional, e Cassiano et al. (2019) verificaram maior incidência de prematuros entre as gestantes com pré-eclâmpsia grave. Apenas Muti et al. (2015) não encontraram associação entre as variáveis avaliadas. 


\section{Discussão}

De acordo com o objetivo da presente revisão integrativa da literatura, a discussão foi embasada nas principais disparidades metodológicas relativas à classificação das SHG e da prematuridade, idade materna das participantes, controle de confundimento e principais resultados dos estudos selecionados.

Para a definição das SHG, utiliza-se como critério de diagnósico de hipertensão, a pressão arterial (PA) de 140/90 mmHg ou mais, em duas ocasiões, no tempo mínimo de quatro horas que se diferenciam em quatro categorias, descritas a seguir: (1) hipertensão crônica: quando surge antes da gestação ou antes da $20^{\mathrm{a}}$ semana de gestação e persiste até a $12^{\mathrm{a}}$ do pósparto; (2) hipertensão na gestação: definida como a hipertensão sem proteinúria que surge após 20 semanas de gestação em uma mulher previamente normotensa; (3) PE/eclâmpsia: determinada pela presença de hipertensão associada a proteinúria ( $\geq 300 \mathrm{mg} / 24 \mathrm{~h}$ ), no entanto, na ausência de proteinúria, diagnóstico é realizado quando a hipertensão estiver acompanhada de qualquer um dos sinais e sintomas relatados a seguir: cefaleia, distúrbios visuais, dor abdominal, trombocitopenia ou aumento das enzimas hepáticas. A eclâmpsia é ocorrência de convulsões na ausência de outros motivos, em gestantes com PE. A síndrome HELLP é conhecida como uma das formas mais graves PE que possui sinais de hemólise, elevação das enzimas hepáticas e baixa contagem de plaquetas e sintomas de dor no quadrante superior direito, mal-estar generalizado, náuseas e vômitos. (4) A $P E$ sobreposta é a manifestação da PE em gestantes com hipertensão (ACOG, 2013; 2019).

Em relação às diretrizes para classificação das SHG, apesar de já existirem algumas que são utilizadas em âmbito internacional (National High Blood Pressure Education Program (NHBPEP, 2000), Sociedade Internacional de Estudos de Hipertensão na Gestação (Brown et al., 2001), American College of Obstetrician and Gynecologist (ACOG, 2002) e a International Statistical Classification of Diseases and Related Health Problems (CID-10)), verificou-se que dos estudos selecionados somente Mora et al. (2010) e Roberts et al. (2005) avaliaram todos os componentes das SHG com base nas diretrizes da NHBPEP (2000) e da Sociedade Internacional de Estudos de Hipertensão na Gestação, respectivamente. Homer et al. (2008) avaliaram a hipertensão gestacional, pré-eclâmpsia com proteinúria e pré-eclâmpsia sem proteinúria e utilizaram as diretrizes da CID-10. Enquanto os demais estudos se basearam em outras diferentes diretrizes não reconhecidas internacionalmente. Destacase que a classficação das SHG com base em diferentes diretrizes resulta alterações na magnitude das medidas de associação, além de dificultar as comparações entre os resultados dos estudos (Homer et al., 2008, Kongwattanakul et al., 2018).

No que diz respeito à definição de prematuridade, a Organização Mundial de Saúde (OMS) em 1977 definiu classicamente esse desfecho quando o nascimento ocorre antes da 37 semana de gestação (entre 140 a 259 dias de gestação) com base no primeiro dia da última menstruação (DUM). A maioria dos estudos selecionados para compor essa revisão (Oliveira et al. (2006), Onyiriuka e Okolo (2007); Homer et al. (2008); Bokhari et al. (2014); Muti et al. (2015) e Cassiano et al. (2019)) utilizou a classificação supracitada. Enquanto, Roberts et al. (2005) e Mora et al. (2010) utilizaram diferentes pontos de corte. Embora o estudo conduzido por Mora et al. (2010) tenha sido realizado antes da publicação do Relatório de Ação Global da Prematuridade da OMS em 2012, observou-se que os autores utilizaram essa classificação (<28; 28 a 32 e 33 a 36 semanas gestacionais). É importante destacar que a avaliação da prematuridade em categorias é de extrema importância, dado que a idade gestacional é inversamente proporcional ao risco de complicações neonatais, ao aumento dos cuidados e à mortalidade neonatal (Phillips et al., 2017; Vanin et al., 2020).

Outro ponto díspare foi a idade das mulheres avaliadas dado que os estudos selecionados avaliaram diferentes faixas etárias. Ainda não se sabe ao certo se a idade materna é um fator de risco independente com efeito direto na prematuridade, ou se age indiretamente associada a fatores de confundimento, tais como as doenças crônicas ou fatores sociodemográficos (Oliveira et al., 2016). Todavia, alguns autores avaliaram que a associação entre as SHG e a prematuridade, quando ocorrida no grupo de adolescentes, pode ser parcialmente explicada pelas maiores taxas de primigestas, reduzido número de consultas de pré-natal, início tardio da assistência, seja por não ter conhecimento da própria gravidez, vergonha de estar grávida ou gestação indesejada 
(Morais et al., 2013), baixa renda familiar (Furtado et al., 2016), baixa escolaridade (Azevedo et al., 2015; Furtado et al., 2016) e dificuldade de acesso aos serviços de saúde (Furtado et al., 2016). Ao passo que, entre as mulheres com idade acima de 35 anos, essa associação pode ser parcialmente explicada pelo processo de senescência ovariana, aumento da frequência de doenças crônicas (Alves et al., 2017; Lima et al., 2018), início tardio da assistência ao pré-natal (Alves et al., 2017).

Em relação ao controle de fatores de confundimento, dentre os estudos incluídos nesta revisão integrativa, quatro deles (Onyiriuka \& Okolo, 2007; Mora et al., 2010; Bokhari et al., 2014; Cassiano et al., 2019) utilizaram dados advindos de prontuários, o que pode acarretar viés de informação e dificuldade de controle de possíveis fatores de confundimento por falta de importantes variáveis tais como, idade das gestantes, antecedentes obstétricos e clínicos, gestações múltiplas, tipo de parto, histórico de SHG, paridade (Ferrero et al., 2016; Heino et al., 2016). No entanto, cabe ressaltar que apenas o artigo conduzido por Homer et al. (2008) controlou os resultados pela paridade.

Em relação à associação entre as SHG e a prematuridade, o estudo transversal conduzido por Roberts et al. (2005) com 24.517 gestantes hipertensas verificou associação entre todas as categorias que compõem as SHG (hipertensão crônica, hipertensão gestacional, hipertensão crônica sobreposta à pré-eclâmpsia, pré-eclâmpsia e eclâmpsia) e a prematuridade. Resultados semelhantes foram observados no estudo caso-controle conduzido por Onyiriuka \& Okolo (2007) com 1.259 casos (gestantes com diagnóstico de hipertensão transitória, PE severa, eclâmpsia, hipertensão crônica ou hipertensão crônica sobreposta à pré-eclâmpsia) e 804 controles (gestantes normotensas).

$\mathrm{Na}$ coorte conduzida por Homer et al. (2008) com 3.345 gestantes, os autores observaram que os recém-nascidos de mulheres com PE com proteinúria tiveram chance aumentada de prematuridade em relação às mulheres com PE sem proteinúria, quando controlado pela paridade. Bokhari et al. (2014), ao conduzirem um estudo transversal com 100 gestantes (50 normotensas e 50 com PE/eclâmpsia), também apuraram que a prevalência de prematuridade foi maior entre as gestantes com PE com proteinúria e edema. De forma semelhante, Mateus et al. (2017), ao analisarem os dados de uma coorte prospectiva multicêntrica de gestantes diagnosticadas com PE e proteinúria maciça $(>5 \mathrm{~g} / 24 \mathrm{~h})$ comparadas às gestantes com PE com diferentes níveis de proteinúria, observaram que as mulheres com PE e proteinúria maciça apresentaram pressão arterial mais elevada, dor epigástrica, aumento da creatinina e ácido úrico séricos antes do parto, menor escore de Apgar aos 5 minutos dos recém-nascidos e mais de $80 \%$ de partos ocorreram com menos de 34 semanas gestacionais.

Conforme observado por Mora et al. (2010), a prematuridade foi superior apenas entre as mulheres com PE/eclâmpsia em relação aos demais grupos estudados. Os autores justificaram os seus resultados com base na alta taxa de cesarianas (52\%) realizadas em mulheres com PE/eclâmpsia com o intuito de reduzir os riscos de morbimortalidade materna e fetal. De forma semelhante, Cassiano et al. (2019), ao conduzirem um estudo transversal com 157 gestantes com pré-eclâmpsia grave (PEG), observaram que 48,8\% dos neonatos nasceram prematuros. Nesse caso, torna-se oportuno citar, que o Ministério da Saúde por meio do Manual Técnico da Gestação de Alto Risco indica a interrupção da gestação em mulheres PEG com idade gestacional maior que 34 semanas como estratégia terapêutica para desfechos adversos maternos e fetais (Brasil, 2012).

No estudo conduzido por Oliveira et al. (2006), observou-se risco superior de prematuridade $(\mathrm{RR}=1,36)$ entre as gestantes com hipertensão crônica comparado às com hipertensão gestacional. Ao analisarem os dados de coorte com 4.892 gestantes. Todavia, Ray et al. (2001) ao analisarem os dados de um coorte em que das 1.948 mulheres, 864 tinham algum tipo de síndrome hipertensiva e ao compararem mulheres com hipertensão gestacional com as demais categorias das síndromes, observaram que os neonatos de gestantes com hipertensão crônica não apresentaram risco aumentando de prematuridade. Dentre os estudos selecionados, apenas Rojas Feria et al. (2009), em estudo transversal com 99 mulheres, com idade média de 30 anos e, em sua maioria, primíparas, avaliaram a associação entre a síndrome HELLP e a prematuridade. Os autores verificaram que $80 \%$ dos partos foram prematuros, com média de 33 semanas gestacionais, nascidos em sua maioria por cesariana (79\%). 
Semelhantemente, Rahmayanti e Nurdiati (2017), em uma coorte com 296 mulheres com PEG, verificaram que o risco de baixo peso ao nascer, asfixia neonatal, nascimento prematuro e cesariana foi superior entre as mulheres com síndrome HELLP.

Dentre os nove estudos selecionados para a presente revisão integrativa, somente o estudo transversal conduzido por Muti et al. (2015), com 56 mulheres com hipertensão gestacional, não encontrou associação entre as SHG e a prematuridade. Tal resultado pode ser parcialmente explicado pelo pequeno tamanho amostral desse estudo.

\section{Considerações Finais}

Em suma, mesmo com a presença de inúmeras divergências metodológicas entre os estudos selecionados, foi observada a associação entre as SHG e a prematuridade em grande parte dos estudos selecionados. Tais resultados demonstraram que a valorização da assistência ao pré-natal se faz necessário às gestantes diagnosticadas com as síndromes hipertensivas, a fim de minimizar ou evitar as complicações neonatais resultantes de tal condição clínica, sobretudo a prematuridade.

Desta forma se faz oportuno a realização de novas estudos com essa temática com base nas diretrizes internacionais existentes para a definição das SHG e da prematuridade.

\section{Referências}

Acien P. (1986). Hypertension and pregnancy: A therapeutic approach Farmacoterapia; 3314-3320.

ACOG. (2002). American College of Obstetricians and Gynecologists. Obstetricians, A. C. of, \& Gynecologists Diagnosis and management of preeclampsia and eclampsia. Obstet. Gynecol., 99, 159-167.

ACOG. (2013). American College of Obstetricians and Gynecologists. Hypertension in pregnancy. Report of the American College of Obstetricians and Gynecologists' task force on hypertension in pregnancy. Obstetrics and gynecology, 122(5), 1122-1131.

ACOG. (2019). American College of Obstetricians and Gynecologists. ACOG Practice Bulletin No. 202: Gestational Hypertension and Preeclampsia. Obstetrics \& Gynecology, 133(1), e1-e25. https://doi.org/10.1097/AOG.0000000000003018

Alves, N. C. C., Feitosa, K. M. A., Mendes, M. E. S., \& Caminha, M. F. C. (2017). Complications in pregnancy in women aged 35 or older. Revista Gaucha de Enfermagem, 38(4), e2017-2042. https://doi.org/10.1590/1983-1447.2017.04.2017-0042

Antunes, M. B., Demitto, M. de O., Gravena, A. A. F., Padovani, C., \& Pelloso, S. M. (2017). Síndrome hipertensiva e resultados perinatais em gestação de alto risco. Revista Mineira de Enfermagem, 21, 1-6. https://doi.org/10.5935/1415-2762.20170067

Azevedo, W. F. de, Diniz, M. B., Fonseca, E. S. V. B. da, Azevedo, L. M. R. de, Evangelista, C. B., Azevedo, W. F. de, Diniz, M. B., Fonseca, E. S. V. B. da, Azevedo, L. M. R. de, \& Evangelista, C. B. (2015). Complicações da gravidez na adolescência: Revisão sistemática da literatura. Einstein (São Paulo), 13(4), 618-626. https://doi.org/10.1590/S1679-45082015RW3127

Barton, J. R., Barton, L. A., Istwan, N. B., Desch, C. N., Rhea, D. J., Stanziano, G. J., \& Sibai, B. M. (2011). Elective delivery at 340/7 to 366/7 weeks' gestation and its impact on neonatal outcomes in women with stable mild gestational hypertension. American Journal of Obstetrics and Gynecology, 204(1), 44.e1-5. https://doi.org/10.1016/j.ajog.2010.08.030

Blencowe, H., Cousens, S., Chou, D., Oestergaard, M., Say, L., Moller, A. B. \& Lawn, J. (2013). Born too soon: the global epidemiology of 15 million preterm births. Reproductive health, 10(1), 1-14. https://doi.org/10.1186/1742-4755-10-S1-S2

Bokhari, Z. H., Yasoob, M. U. H. A. M. M. A. D., Intesar, A. Y. E. S. H. A., \& Haq, M. F. U. (2014). Neonatal outcome in patients with preeclampsia. Pak J Med Health Sci, 8(4), 970-972.

Brandi, L. D. de A., Rocha, L. R., Silva, L. S., Bretas, L. G., Rodrigues, M. A., \& Araújo, S. T. H. de. (2020). Fatores de risco materno-fetais para o nascimento pré-termo em hospital de referência de Minas Gerais. 30(0), 41-47. https://doi.org/10.5935/2238-3182.v30supl.4.06

Brasil (2012). Gestação de Alto Risco. Manual Técnico. Departamento de Ações Programáticas Estratégicas. Série A. Normas e Manuais Técnicos (5ed). http://bvsms.saude.gov.br/bvs/publicacoes/manual_tecnico_gestacao_alto_risco.pd>

Brasil (2014). Diretrizes Metodológicas: Elaboração de revisão sistemática e metanálise de estudos observacionais comparativos sobre fatores de risco e prognóstico. Ministério da Saúde, Secretária de Ciência e Insumos Estratégicos. Departamento de Ciência e Tecnologia. (1 ${ }^{\circ}$ ed).

Braunthal, S., \& Brateanu, A. (2019). Hypertension in pregnancy: Pathophysiology and treatment. SAGE open medicine (7) 1-15, 2050312119843700. https://doi.org/10.1177/2050312119843700

Brown, M. A., Lindheimer, M. D., de Swiet, M., Van Assche, A., \& Moutquin, J. M. (2001). The classification and diagnosis of the hypertensive disorders of pregnancy: Statement from the International Society for the Study of Hypertension in Pregnancy (ISSHP). Hypertension in Pregnancy, 20(1), 9-14.

https://doi.org/10.3109/10641950109152635 
Cassiano, A. do N., Vitorino, A. B. F., Silva, M. de L. C. da, Nóbrega, C. S. M. de H. da, Pinto, E. S. G., \& Souza, N. L. de. (2019). Desfechos perinatais de gestantes com pré-eclampsia grave: Estudo transversal. Online Braz. j. Nurs. (Online). (18)4. https://doi.org/10.17665/1676-4285.2019v18n4

Chaim, S. R. P., Oliveira, S. M. J. V. de, \& Kimura, A. F. (2008). Hipertensão arterial na gestação e condições neonatais ao nascimento. Acta Paulista de Enfermagem, 21(1), 53-58. https://doi.org/10.1590/S0103-21002008000100008

CID 10. (2004). National Centre for Classification in Health. International statistical classification of diseases and related health problems, Australian modification (ICD-10-AM). Sydney: National Centre for Classification in Health, University of Sydney, 2004. 10th revision.

Cruz, S. S. da, Costa, V. S., Santos, J. M. da M., Gomes, T. A. O., Brandão, I. R., Fonseca, C. H. A., Vilasboas, S. W. S. L., Silva, R. B., Filho, S.A.A S. G., Souza, L. M., Figueiredo, A. C. M. G., \& Batista, J. (2018). Prematuridade ao nascer, hipertensão materna e outros fatores associados: um estudo de casocontrole na região do Vale do São Francisco. Revista Brasileira de Pesquisa Em Saúde/Brazilian Journal of Health Research, 20(4), 113-119.

Cunningham, F., Mac Donald, P., Gant, N., Leveno, K., \& Gilstrap, L. (1993). Hypertensive disorders in pregnancy. In: Cunningham, MacDonald P,(eds) Williams Obstetrics. 19th, 819-852.

Ferrero, D. M., Larson, J., Jacobsson, B., Renzo, G. C. D., Norman, J. E., Jr, J. N. M., D’Alton, M., Castelazo, E., Howson, C. P., Sengpiel, V., Bottai, M., Mayo, J. A., Shaw, G. M., Verdenik, I., Tul, N., Velebil, P., Cairns-Smith, S., Rushwan, H., Arulkumaran, S., \& Simpson, J. L. (2016). Cross-Country Individual Participant Analysis of 4.1 Million Singleton Births in 5 Countries with Very High Human Development Index Confirms Known Associations but Provides No Biologic Explanation for 2/3 of All Preterm Births. PLOS ONE, 11(9), e0162506. https://doi.org/10.1371/journal.pone.0162506

Furtado, É. Z. L., Gomes, K. R. O., \& Gama, S. G. N. da. (2016). Access to childbirth care by adolescents and young people in the Northeastern region of Brazil. Revista de Saúde Pública, 50(23) 1-6. https://doi.org/10.1590/S1518-8787.2016050005396

Heino, A., Gissler, M., Hindori-Mohangoo, A. D., Blondel, B., Klungsøyr, K., Verdenik, I., Mierzejewska, E., Velebil, P., Ólafsdóttir, H. S., Macfarlane, A., Zeitlin, J., \& Committee, E.-P. S. (2016). Variations in Multiple Birth Rates and Impact on Perinatal Outcomes in Europe. PLOS ONE, 11(3), e0149252. https://doi.org/10.1371/journal.pone.0149252

Homer, C. S., Brown, M. A., Mangos, G., \& Davis, G. K. (2008). Non-proteinuric pre-eclampsia: A novel risk indicator in women with gestational hypertension. Journal of Hypertension, 26(2), 295-302. https://doi: 10.1097/HJH.0b013e3282f1a953

Kongwattanakul, K., Saksiriwuttho, P., Chaiyarach, S., \& Thepsuthammarat, K. (2018). Incidence, characteristics, maternal complications, and perinatal outcomes associated with preeclampsia with severe features and HELLP syndrome. International Journal of Women's Health, 10, 371-377. https://doi: 10.2147/IJWH.S16856

Lima, A. de A., Tenório, M. C. dos S., Dutra, T. A., Bomfim, I. C., \& Oliveira, A. C. M. de. (2018). Caracterização de recém-nascidos prematuros nascidos em maternidade de referência de alto risco de Maceió, Alagoas. Gep News, 1(1), 32-37.

Lima, J. P., do Nascimento Veras, L. L., da Silva Pedrosa, É. K. F., Oliveira, G. D. S. C., \& Guedes, M. V. C. (2018). Perfil socioeconômico e clínico de gestantes com Síndrome Hipertensiva Gestacional. Rev Rene, 19, 1-7. 19, e3455. https://doi.org/10.15253/2175-6783.2018193455

Lu, C.-Q., Lin, J., Yuan, L., Zhou, J.-G., Liang, K., Zhong, Q.-H., Huang, J.-H., Xu, L.-P., Wu, H., Zheng, Z., Ping, L.-L., Sun, Y., Li, Z.-K., Liu, L., Lyu, Q., \& Chen, C. (2018). Pregnancy induced hypertension and outcomes in early and moderate preterm infants. Pregnancy Hypertension, $14,68-71$. https://doi.org/10.1016/j.preghy.2018.06.008

Martin, J. N., Rinehart, B. K., May, W. L., Magann, E. F., Terrone, D. A., \& Blake, P. G. (1999). The spectrum of severe preeclampsia: Comparative analysis by HELLP (hemolysis, elevated liver enzyme levels, and low platelet count) syndrome classification. American Journal of Obstetrics and Gynecology, 180(6), 1373-1384. https://doi.org/10.1016/S0002-9378(99)70022-0

Mateus, J., Newman, R., Sibai, B. M., Li, Q., Barton, J. R., Combs, C. A., Guzman, E., Boggess, K. A., Gyamfi, C., von Dadelszen, P., \& Woelkers, D. (2017). Massive Urinary Protein Excretion Associated with Greater Neonatal Risk in Preeclampsia. AJP Reports, 7(1), e49-e58. https://doi.org/10.1055/s-0037-1601866

Moher, D., Liberati, A., Tetzlaff, J., Altman, D. G., \& PRISMA Group. (2009). Prefered reporting items for systematic reviews and meta-analyses: The PRISMA statement. PLoS Medicine, 6(7), e1000097.

Mora, P., Gobernado, J. A., Pérez-Milán, F., \& Cortés, S. (2010). Estados hipertensivos del embarazo: Resultados perinatales. Clínica e Investigación en Ginecología y Obstetricia, 37(2), 56-62. https://doi.org/10.1016/j.gine.2009.03.008

Morais, F. M., Novais, J. M., Silveira, M. C. A., Araujo, M. J. A. R., Melo, S. M., \& Gontijo, L. S. (2013). Perfil clínico-epidemiológico e repercussões perinatais em portadoras de síndrome hipertensiva gestacional: uma revisão. Revista Eixo, 2(1), 69-82. https://doi.org/10.19123/eixo.v2i1.103

Muti, M., Tshimanga, M., Notion, G. T., Bangure, D., \& Chonzi, P. (2015). Prevalence of pregnancy induced hypertension and pregnancy outcomes among women seeking maternity services in Harare, Zimbabwe. BMC Cardiovascular Disorders, 15(1), 111. https://doi.org/10.1186/s12872-015-0110-5

NHBPEP (2000). Report of the National High Blood Pressure Education Program Working Group on High Blood Pressure in Pregnancy. American Journal of Obstetrics and Gynecology, 183(1), s1-s22. https://doi.org/10.1067/mob.2000.107928

Oliveira, A. C. M. de, Santos, A. A., Bezerra, A. R., Barros, A. M. R. de, Tavares, M. C. M., Oliveira, A. C. M. de, Santos, A. A., Bezerra, A. R., Barros, A. M. R. de, \& Tavares, M. C. M. (2016). Fatores Maternos e Resultados Perinatais Adversos em Portadoras de Pré-eclâmpsia em Maceió, Alagoas. Arquivos Brasileiros de Cardiologia, 106(2), 113-120. https://doi.org/10.5935/abc.20150150

Oliveira, C. A. de, Lins, C. P., Sá, R. A. M. de, Netto, H. C., Bornia, R. G., Silva, N. R. da, \& Amim Junior, J. (2006). Síndromes hipertensivas da gestação e repercussões perinatais. Revista Brasileira de Saúde Materno Infantil, 6(1), 93-98. https://doi.org/10.1590/S1519-38292006000100011

OMS. (1977). WHO: Recommended definitions, terminology and format for statistical tables related to the perinatal period and use of a new certificate for cause of perinatal deaths. Modifications recommended by FIGO as amended October 14, 1976. Acta Obstetricia Et Gynecologica Scandinavica, 56(3), $247-253$. 
OMS. (2014). Organização Mundial de Saúde. Recomendação da OMS para prevenção e tratamento da pré-eclâmpsia e eclâmpsia. 48p.

Onyiriuka, A. N., \& Okolo, A. A. (2007). Neonatal Morbidity Pattern In Infants Born In Benin City To Nigerian Mothers With Hypertensive Disorders In Pregnancy. Nigerian Journal of Clinical Practice, 10(4), 294-299-299.

Pereira, S. S. M., Jesus, M. N., Rodrigues, J. M., Miranda, F. C., Ribeiro, I. P., \& Silva, A. D. (2018). Perfil de Gestantes Acometidas de Parto Prematuro em uma Maternidade Pública. Revista de Pesquisa: Cuidado é Fundamental Online, 10(3), 758-763. https://doi.org/10.9789/2175-5361.2018.v10i3.758-763

Phillips, C., Velji, Z., Hanly, C., \& Metcalfe, A. (2017). Risk of recurrent spontaneous preterm birth: A systematic review and meta-analysis. BMJ Open, 7(6) . e015402. https://doi.org/10.1136/bmjopen-2016-015402

Purisch, S. E., \& Gyamfi-Bannerman, C. (2017). Epidemiology of preterm birth. Seminars in Perinatology, 41(7), 387-391. https://doi.org/10.1053/j.semperi.2017.07.009

Ray, J. G., Burrows, R. F., Burrows, E. A., \& Vermeulen, M. J. (2001). MOS HIP: McMaster outcome study of hypertension in pregnancy. Early Human Development, 64(2), 129-143. https://doi.org/10.1016/S0378-3782(01)00181-5

Rahmayanti, S., \& Nurdiati, D. S. (2017). HELLP syndrome in severe preeclampsia: The perinatal outcomes. Pregnancy Hypertension: An International Journal of Women's Cardiovascular Health, 7, 61. https://doi.org/10.1016/j.preghy.2016.10.017

Roberts, C. L., Ford, J. B., Henderson-Smart, D. J., Algert, C. S., \& Morris, J. M. (2005). Hypertensive disorders in pregnancy: A population-based study. Medical Journal of Australia, 182(7), 332-335. https://doi.org/10.5694/j.1326-5377.2005.tb06730.x

Rojas Feria, P., Losada Martínez, A., \& Miranda Guisado, M. L. (2009). Complicaciones neonatales del síndrome HELLP. Anales de Pediatría, 70(2), 159-163. https://doi.org/10.1016/j.anpedi.2008.08.023

Scime, N. V., Chaput, K. H., Faris, P. D., Quan, H., Tough, S. C., \& Metcalfe, A. (2020). Pregnancy complications and risk of preterm birth according to maternal age: A population-based study of delivery hospitalizations in Alberta. Acta Obstetricia et Gynecologica Scandinavica, 99(4), 459-468. https://doi.org/10.1111/aogs.13769

SEGO (1988). Grupo “'Estados Hipertensivos del Embarazo”'. Documentos de Consenso. Sociedade Espanhola de Obstetricia Ginecologia. 45-78.

Shah, S., \& Gupta, A. (2019). Hypertensive Disorders of Pregnancy. Cardiology Clinics, 37(3), 345-354. https://doi.org/10.1016/j.ccl.2019.04.0087

Sousa, M. G., Lopes, R. G. C., Rocha, M. L. T. L. F., Lippi, U. G., Costa, E. S., \& Santos, C. M. P. (2019). Epidemiology of artherial hypertension in pregnants. einstein (São Paulo), 18, eAO4682. https://doi.org/10.31744/einstein_journal/2020AO4682

Sousa, L. M. M. S., Marques-Vieira, C. M. A., Severino, S. S., \& Antunes, A. V. (2017). Metodologia de Revisão Integrativa da Literatura em Enfermagem. 17 -26. http://repositorio-cientifico.essatla.pt/handle/20.500.12253/1311

Vanin, L. K., Zatti, H., Soncini, T., Nunes, R. D., \& Siqueira, L. B. S. de. (2020). Maternal and Fetal Risk Factors Associated with Late Preterm Infants. Revista Paulista de Pediatria, 38, e2018136. https://doi.org/10.1590/1984-0462/2020/38/2018136

Viana, T. P., Andrade, I. S. N. de, Lopes, A. N. M., Viana, T. P., Andrade, I. S. N. de, \& Lopes, A. N. M. (2014). Desenvolvimento cognitivo e linguagem em prematuros. Audiology - Communication Research, 19(1), 1-6. https://doi.org/10.1590/S2317-64312014000100002

Welt, S. I., \& Crenshaw, M. C. (1978). Concurrent hypertension and pregnancy. Clinical Obstetrics and Gynecology, 21(3), 619-648. https://doi.org/10.1097/00003081-197809000-00003 